ORIGINAL ARTICLE

\title{
Master's performance in the New York City Marathon 1983-1999
}

\author{
P Jokl, P M Sethi, A J Cooper
}

Br J Sports Med 2004;38:408-412. doi: 10.1136/bjsm.2002.003566

See end of article for authors' affiliations

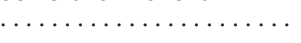

Correspondence to: Professor Jokl, Department of Orthopaedics, Yale University, Yale Physicians Building, 800 Howard Avenue, New Haven, CT 06512, USA; peter. jokl@yale.edu

Accepted 16 June 2003
Background: Physical activity in older people is believed to slow down the natural aging process through its effects on disuse atrophy.

Objectives: To show that elite master (age above 50) male and female athletes, as a group, have improved their running times over the last two decades at a greater rate than their younger counterparts.

Methods: Running time, age, and sex of all 415000 runners in the New York City Marathon from 1983 to 1999 were examined using linear regression analysis.

Results: The number of master participants increased at a greater rate than their younger counterparts $(p<0.05)$. Running times for the top 50 male and female finishers over the past two decades showed significantly greater improvement in the master groups than in the younger age groups $(p<0.001)$.

Conclusions: Participation in the New York City Marathon is increasing at a higher rate in the master groups than in other age groups. Male and female masters continued to improve running times at a greater rate than the younger athletes, whose performance levels have plateaued. This is the largest study to compare master athletic performance with younger counterparts and men with women.
A s we continue to enjoy greater longevity, doctors will inevitably be challenged to provide appropriate medical care and advice to our aging population. ${ }^{12}$ Many of the changes in health status previously thought be a consequence of normal aging have been shown to be a result of a sedentary lifestyle. $^{3-5}$

A sedentary lifestyle is the most prevalent modifiable risk factor for development of coronary heart disease. ${ }^{5}$ According to the American Heart Association (2003 statistics, AHA), $51 \%$ of men and $48 \%$ of women aged 55-64 have cardiovascular disease. The incidence increases to $65 \%$ in women and men aged 65-74. Aging is further associated with decreased muscular strength and endurance leading to a declining functional capacity and quality of life. ${ }^{6}$ The most dramatic aspect of this decline is probably not the result of aging but the inactivity associated with aging. Regular physical activity can reduce the risk of all cause mortality by about $25 \%$ and increase life expectancy by up to two years. ${ }^{7}$ Despite the benefits of exercise, only $20-30 \%$ of all adults engage in vigorous activity on a regular basis, and a staggering $25 \%$ are totally inactive. ${ }^{2} 7$ Furthermore, physician knowledge of performance potential in older athletes is quite limited.

Studies describing age related decreases in performance suggest that athletic performance falls at a linear rate until the age of 70, at which point the decreases become more precipitous, specifically in running events. These performance profiles physiologically correlate with muscle strength, which peaks at the end of the third decade of life, and starts to decline at $50 .^{7}$ It has been shown that a person's level of activity modifies the rate and degree of decline in muscle strength, which in turn may blunt the effects of aging on physical performance. ${ }^{8}$

In addition to age related changes, current attention has also focused on sex related differences in aging and its physiology. Women, for example, showed greater age related declines than men in long and short duration swimming events. ${ }^{1}$ However, there are limited data describing the performance differences between aging men and women. ${ }^{8}$

The recommendations for therapeutic exercise in our aging population are in a state of evolution. ${ }^{26}{ }^{9}$ The performance of master athletes (aged above 50), along with the exponential increase in master participation in sporting events, continues to push the performance envelope that had once been applied to this age group. ${ }^{7}$ A careful study of people who age successfully from a physical performance standpoint may elucidate methods and mechanisms that doctors can apply to all patients to develop a more uniform healthcare approach. One such group is the master athlete. To the best of our knowledge, there are no cross sectional studies on the performance of master athletes that evaluate their rate of performance improvement over the past two decades.

In this study we evaluated the performances of the top 50 male and top 50 female finishers by age categories in the New York City (NYC) Marathon from 1983 to 1999. Our hypothesis was that elite master runners have improved their mean performance times as a group over the study period, and at a faster rate than elite younger athletes. Furthermore, we expected these trends to be significant for both sexes.

\section{METHODS}

We examined the running time, age, and sex of all runners in the NYC Marathon from 1983 through 1999. During this period, there were 415000 runners.

We defined master athletes as those equal to or older than 50. There is no consensus in the literature on the age definition of a master athlete, with some studies including athletes older than 30 as masters and others setting the limit at 60 and older.

We separated all qualifying runners into groups based on their age and sex: $\mathrm{M}=$ male and $\mathrm{F}=$ female; 1 was used for the decade from 10 to 19,2 for 20-29, 3 for 30-39, 4 for 40 49,5 for $50-59,6$ for $60-69$, and 7 for $70-79$. Thus in our data analysis " $\mathrm{M}$ age group $\mathrm{l}$ " comprises all male runners aged 10-19, and "F age group 2" comprises all female runners aged 20-29, and so on. There is a set of data for each of these groups for every year from 1983 to 1999 . This data orientation allowed evaluation of running performances for different age groups over the period reviewed. 
For each age bracket, the mean performance times for all finishers, as well as the top 50 male and female finishers, were evaluated for each year.

Linear regression was performed on all groups to look for trends in age related performance over the past 17 years. Linear regression was also performed on all groups to look for age and sex related trends in participation over the last 17 years. When evaluating the top 50 finishers, we did not remove runners that qualified in more than one year. There was a small, statistically insignificant number of repeat runners during the 17 year study period that ranged from 1 to $2.5 \%$ of the top 50 qualifiers in the men's groups 5 and 6 , and the women's group 4,5, and 6. All data were transferred from separate files in Excel (Microsoft Excel 2000) to the statistical program SPSS (SPSS, Inc 2000). All the information for the 415000 runners was compiled and sorted, with subsequent linear regression analysis. Linear regression analysis is the appropriate statistical test to correlate behaviour between two variables and to test the significance of such a correlation. All data were reproducible using SPSS and subsequent linear regression.

\section{RESULTS}

\section{Participation}

Participation in the NYC marathon increased over the period 1983-1999. In 1983, 14546 runners completed the marathon, and by 1999, this number had increased $119 \%$ to 31791 (table 1). During this period, there were no restrictions based on sex and no age based limitations above 18. Entry was based on a lottery system. There has been a linear decline in the ratio of male to female runners, from 5.6:1 in 1983 to 2.47:1 in 1999, reflecting the increase in female applications.

Figure 1 shows the ratio of participation over time. The decline in fig 1 regresses to the line: ratio $=-0.202 \times($ year $)$ +406.65 . This indicates the decrease in the ratio of male to female runners over the last 17 years $(p<0.001)$.

All age segments for both sexes show significantly increased participation over the past 17 years $(p<0.001)$, except for male runners in age group 1. Table 2 presents participation differences by age group and sex.

The greatest absolute increases in male participation were in age groups 3,4, and 5. The greatest absolute increases in female participation were in age groups $2,3,4$, and 5 . When normalised for age group participation size, the older age groups for both male and female runners showed the largest relative increase in participation.

Table 1 Number of runners in the New York City Marathon over the study period

\begin{tabular}{lllll}
\hline \multicolumn{5}{c}{ Runners per year } \\
Year & Men & Women & Total & M/F ratio \\
\hline 1983 & 12341 & 2205 & 14546 & 5.60 \\
1984 & 12198 & 2395 & 14593 & 5.09 \\
1985 & 13417 & 2481 & 15898 & 5.41 \\
1986 & 16394 & 3339 & 19733 & 4.91 \\
1987 & 17557 & 3688 & 21245 & 4.76 \\
1988 & 18379 & 3958 & 22337 & 4.64 \\
1989 & 19966 & 4688 & 24654 & 4.26 \\
1990 & 19278 & 4500 & 23778 & 4.28 \\
1991 & 20596 & 5202 & 25798 & 3.96 \\
1992 & 22370 & 5448 & 27818 & 4.11 \\
1993 & 20785 & 5813 & 26598 & 3.58 \\
1994 & 22757 & 6975 & 29732 & 3.26 \\
1995 & 20285 & 6467 & 26752 & 3.14 \\
1996 & 20750 & 7431 & 28181 & 2.79 \\
1997 & 22022 & 8412 & 30434 & 2.62 \\
1998 & 22587 & 8952 & 31539 & 2.52 \\
1999 & 22629 & 9162 & 31791 & 2.47 \\
\hline
\end{tabular}

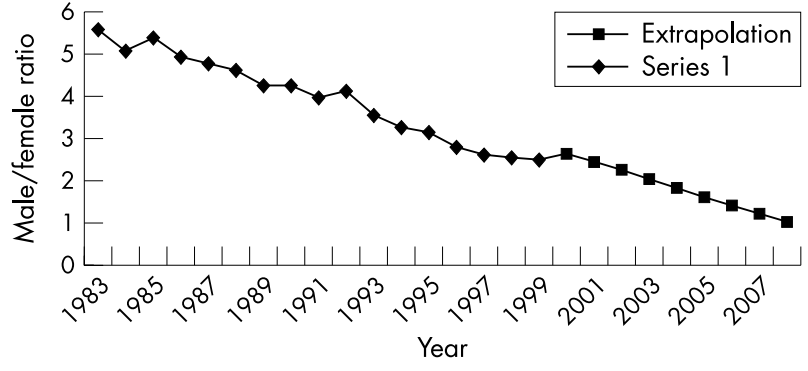

Figure 1 Comparison of the number of male and female runners over time in the New York City Marathon. If this linear pattern continues, the ratio is expected to approach 1 in 2007.

Table 2 Trends in participation in the New York City Marathon in the different age groups

\begin{tabular}{|c|c|c|c|c|}
\hline & $\begin{array}{l}\text { Age } \\
\text { group }\end{array}$ & $\begin{array}{l}\text { Mean yearly } \\
\text { change }\end{array}$ & $\begin{array}{l}\text { Relative } \\
\text { change }\end{array}$ & p Value \\
\hline \multicolumn{5}{|l|}{ Men } \\
\hline & 1 & -1.83 & -0.017 & $<0.001$ \\
\hline & 2 & 27.34 & 0.008 & $<0.001$ \\
\hline & 3 & 78.77 & 0.011 & $<0.001$ \\
\hline & 4 & 83.54 & 0.015 & $<0.001$ \\
\hline & 5 & 78.41 & 0.033 & $<0.001$ \\
\hline & 6 & 22.13 & 0.047 & $<0.001$ \\
\hline & 7 & 2.6 & 0.042 & $<0.001$ \\
\hline \multicolumn{5}{|l|}{ Women } \\
\hline & 1 & 0.268 & 0.010 & $<0.001$ \\
\hline & 2 & 67.88 & 0.045 & $<0.001$ \\
\hline & 3 & 79.6 & 0.038 & $<0.001$ \\
\hline & 4 & 51.36 & 0.041 & $<0.001$ \\
\hline & 5 & 27.19 & 0.068 & $<0.001$ \\
\hline & 6 & 3.82 & 0.060 & $<0.001$ \\
\hline & 7 & 0.95 & 0.102 & $<0.001$ \\
\hline \multicolumn{5}{|c|}{$\begin{array}{l}\text { The age group numbers are: } 1 \text {, the decade from } 10 \text { to } 19 ; 2,20-29 ; 3 \text {, } \\
30-39 ; 4,40-49 ; 5,50-59 ; 6,60-69 ; 7,70-79 \text {. The mean yearly } \\
\text { change is the percentage increase in runners in each age group, a } \\
\text { positive result indicating a net increase and a negative result indicating a } \\
\text { net decrease. The relative change is the percentage change in the net } \\
\text { numbers as they relate to the total number in that age bracket. It is an } \\
\text { adjusted value to correct for the variable number of runners in each age } \\
\text { bracket. }\end{array}$} \\
\hline
\end{tabular}

The greatest relative increase in participation for both male and female runners was in age groups 5, 6, and 7. This indicates that, across age segments, participation continues to increase, but at different rates for age group and sex. There is a relative increase in participation as the age groups get older for both male and female runners. There is a greater relative increase in participation for female runners across all age segments. This underscores the results in table 2, as female participation increased at a greater rate than male participation.

\section{Finishing times}

Tables 3 and 4 list the mean marathon times broken down by age group for the top 50 male and 50 female finishers respectively, from 1983 to 1999 . Changes in the mean times are also listed through linear regression analysis. The "slope" is the change in time (minutes)/year, determined by linear regression. A positive slope corresponds to an increase in the average running time for that age group, and a negative slope corresponds to a decrease in average running time for that age group. The mean running times for all male and female participants increased-that is, were slower-over the time period studied (data not shown). 
Table 3 Mean finishing times broken down by age group for the top 50 male finishers in the New York City Marathon from 1983 to 1999

\begin{tabular}{|c|c|c|c|c|c|c|c|}
\hline & \multicolumn{7}{|c|}{ Age group } \\
\hline & 1 & 2 & 3 & $4^{*}$ & 5 & 6 & $7 \dagger$ \\
\hline 1983 & 202.53 & 136.32 & 140.94 & 157.07 & 179.2 & 217.84 & 310.21 \\
\hline 1984 & 216.17 & 145.93 & 151.9 & 167.16 & 193.66 & 237.97 & 330.44 \\
\hline 1985 & 209.04 & 143.53 & 145.79 & 165.07 & 183.69 & 228.3 & 316.12 \\
\hline 1986 & 210.69 & 141.09 & 144.85 & 161.08 & 178.03 & 216.46 & 310.09 \\
\hline 1987 & 208.34 & 142.08 & 144.19 & 157.96 & 180.14 & 222.52 & 316.7 \\
\hline 1988 & 214.07 & 145.76 & 147.2 & 161.73 & 176.86 & 220.08 & 322.18 \\
\hline 1989 & 211.69 & 144.5 & 145.02 & 157.19 & 176.34 & 214.19 & 325.05 \\
\hline 1990 & 226.04 & 150.21 & 149.48 & 160.45 & 178.13 & 220.54 & 322.05 \\
\hline 1991 & 215.82 & 147.71 & 144.39 & 160.88 & 175.58 & 209.88 & 296.44 \\
\hline 1992 & 227.34 & 146.11 & 145.16 & 157.32 & 173.7 & 211.95 & 299.39 \\
\hline 1993 & 230.21 & 148.58 & 147.08 & 163.52 & 182.97 & 216.85 & 318.66 \\
\hline 1994 & 234.54 & 150.34 & 146.12 & 162.51 & 180.69 & 211.46 & 305.36 \\
\hline 1995 & 230.98 & 145.32 & 147.01 & 164.18 & 182.48 & 215.73 & 303.61 \\
\hline 1996 & 233.15 & 150.25 & 147.76 & 164.13 & 183.27 & 209.25 & 306 \\
\hline 1997 & 240.23 & 150.8 & 149.75 & 163.9 & 182.43 & 207.84 & 306.13 \\
\hline 1998 & 234.01 & 144.23 & 147.06 & 158.94 & 177.2 & 208.85 & 280.37 \\
\hline 1999 & 244.56 & 152.15 & 149.87 & 162.7 & 181.18 & 207.69 & 291.26 \\
\hline Slope & 2.33 & 0.576 & 0.205 & 0.0078 & -0.126 & -1.226 & -1.89 \\
\hline
\end{tabular}

Unless specified $(t)$, the slope is significant $(p<0.05)$. Slope represents trend in minutes/year.

*Not all subgroups had 50 finishes.

†Slope not statistically significant.

The best 50 female runners, like the male runners, in age groups 1 and 2 showed a significant mean increase in running time $(\mathrm{p}<0.001)$. Female runners in age groups 4,5 , and 6 showed a significant mean decrease in running time $(\mathrm{p}<0.001)$, with the greatest time improvement seen in age group 6 of 3.79 minutes a year (table 4 ).

Figures 2 and 3 show the mean performance times for each age group and sex. The results indicate a significant improvement in time and performance over the last 17 years for the older age groups for both men and women.

In summary, during this period, male runners aged 50-59 improved their running times by about eight seconds ( 0.13 minutes) a year, and female runners in this age group by 2.08 minutes a year. Male runners aged 60-69 improved by 1.23 minutes annually compared with 3.79 minutes annually for female runners $(p<0.05)$. Male runners in the 70-79 age group improved by 1.90 minutes annually $(\mathrm{p}<0.05)$.

\section{DISCUSSION}

This study presents data showing that, as a group, master athletes improved their performance times in the NYC Marathon at a greater rate than younger athletes over the last 17 years. Twenty five years ago, few 60 year old men, much less women, or their doctors would have considered it possible for someone of their age to run 26 plus miles. A more thorough understanding of age related physiological changes and the potential slowing of some of the aging processes through athletic training was achieved in the 1960s. This better understanding coupled with the exponential increase in participation of master athletes in sporting events has led to re-evaluation of the aging process and how it relates to athletic performance.

A review of the literature on the effects of aging on muscle indicates that this process results in loss of muscle mass and a change in the proportions of fibre types. ${ }^{8}{ }^{10}$ There is an increase in the proportion of type I fibres (oxidative, slow

Table 4 Mean finishing times broken down by age group for the top 50 female finishers in the New York City Marathon from 1983 to 1999

\begin{tabular}{|c|c|c|c|c|c|c|}
\hline & \multicolumn{6}{|c|}{ Age group } \\
\hline & 1 & 2 & $3^{*}$ & 4 & 5 & $6 t$ \\
\hline 1983 & 260.85 & 169.3 & 176.94 & 196.92 & 255.95 & 302.38 \\
\hline 1984 & 275.42 & 184.75 & 192.56 & 213.39 & 264.85 & 382.82 \\
\hline 1985 & 278.34 & 182.08 & 182.5 & 203.17 & 257.28 & 344.64 \\
\hline 1986 & 300.69 & 179.89 & 179.85 & 205.38 & 247.48 & 325.29 \\
\hline 1987 & 284.57 & 181.97 & 182.79 & 199.7 & 243.19 & 331.7 \\
\hline 1988 & 301.16 & 183.61 & 183.58 & 206 & 234.93 & 330.51 \\
\hline 1989 & 314.43 & 179.85 & 177.47 & 195.25 & 222.85 & 320.04 \\
\hline 1990 & 311.22 & 195.3 & 181.01 & 203.01 & 231.85 & 308.48 \\
\hline 1991 & 327.98 & 187.7 & 179.77 & 197.88 & 226.58 & 326.05 \\
\hline 1992 & 315.86 & 184.46 & 178.35 & 202.91 & 225.24 & 304.6 \\
\hline 1993 & 304.71 & 190.46 & 181.01 & 203.73 & 232.14 & 310.88 \\
\hline 1994 & 314.95 & 190.29 & 182.21 & 197.82 & 226.5 & 293.88 \\
\hline 1995 & 303.98 & 190.08 & 185.18 & 205.84 & 238.07 & 303.47 \\
\hline 1996 & 304.76 & 189.95 & 182.41 & 198.31 & 227.89 & 294.62 \\
\hline 1997 & 308.57 & 188.93 & 184.71 & 201.03 & 225.2 & 297.07 \\
\hline 1998 & 316.58 & 184.32 & 183.49 & 194.75 & 226.08 & 285.76 \\
\hline 1999 & 302.38 & 193.63 & 185.46 & 198.49 & 224.3 & 288.71 \\
\hline Slope & 2.12 & 0.85 & 0.1 & -0.37 & -2.08 & -3.79 \\
\hline
\end{tabular}

Unless specified $(t)$, the slope is significant $(p<0.05)$. Slope represents trend in minutes/year.

*Not all subgroups had 50 finishes.

†Slope not statistically significant. 


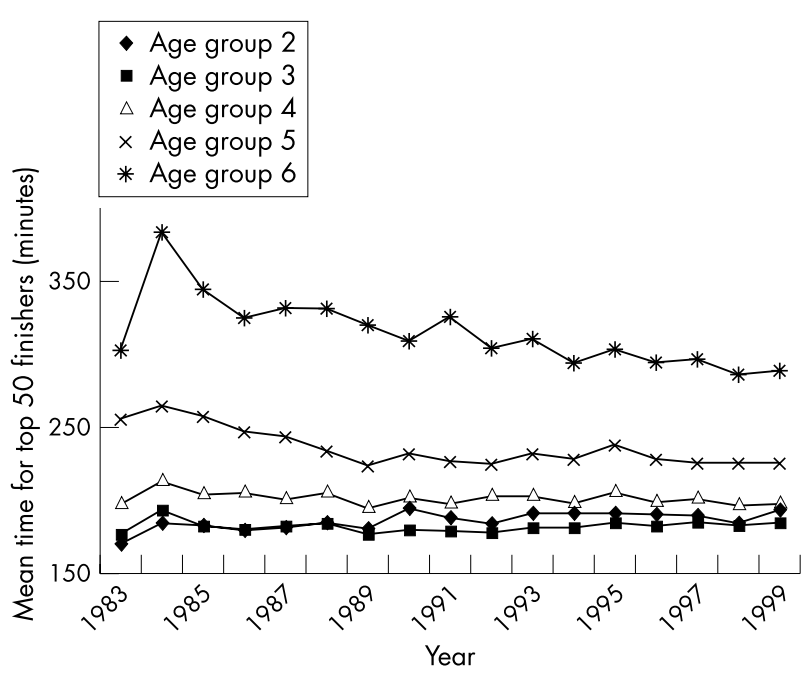

Figure 2 Mean running times of the top 50 female finishers in the NYC Marathon broken down by age group. Runners in age group 2 showed a significant increase in running time $(p<0.001)$. Runners in age groups 4,5 , and 6 showed a significant decrease in running time $(p<0.001)$, with the greatest time improvement seen in age group 6 of 3.79 minutes a year.

twitch), which may be an adaptation to high endurance demands. ${ }^{11}$ Cardiovascular and aerobic capacity $\left(\mathrm{VO}_{2} \mathrm{MAX}\right)$ are diminished with aging. The decrease in $\mathrm{VO}_{2} \mathrm{MAX}$ is due to a decrease in maximal heart rate and not a decrease in maximum stroke volume or oxygenation of the blood in non-sedentary elderly. Thus, the rate of the diminution is modified by physical training. ${ }^{12}$ Pollock et al ${ }^{13}$ also showed maintenance of $\mathrm{VO}_{2} \mathrm{MAX}$, over a 10 year period in master athletes with continued training. Hagberg et $a l^{15}$ further showed that master athletes have a lower vascular resistance and a higher stroke volume than their sedentary counterparts. There are many studies that conclude that master athletes tend to be less depressed, angry, and fatigued than their non-athletic counterparts. ${ }^{2}$

We chose to study elite master athletes because the analyses of running times in the all comers group of master athletes do not reflect the maximal performance levels attainable. Our overall data showed an increased mean finishing time for all comers in the masters group (data not shown). We believe that this actually reflects the increasing number of competitors in all age groups at various levels of athletic fitness and ability. Over the last two decades, there is a higher proportion of outlier times-that is, 7-8 hourswhich skew the average times towards a slower performance (data not shown). A more detailed analysis of the top 50 male and top 50 female master finishers, in contrast, shows a significant improvement in their performance over time (tables 3 and 4). We see these athletes as a better representation of the best performance times attainable at a given age level. The top finishers among the master athletes are improving their performance as a group at a significantly greater rate than their younger counterparts $(p<0.05)$. The performance of the 20-30 and 30-40 age groups has actually plateaued, with no improvement in running time for the overall group being observed for the time period studied.

Our data reflect the potential for improvement of the general health of our aging population. It is not surprising that the number of participating master athletes continues to rise as there is a general trend towards increasing numbers of the aging population who are in good health and physically able to participate in these types of strenuous competitions. The modern advances of medical care and proper nutrition

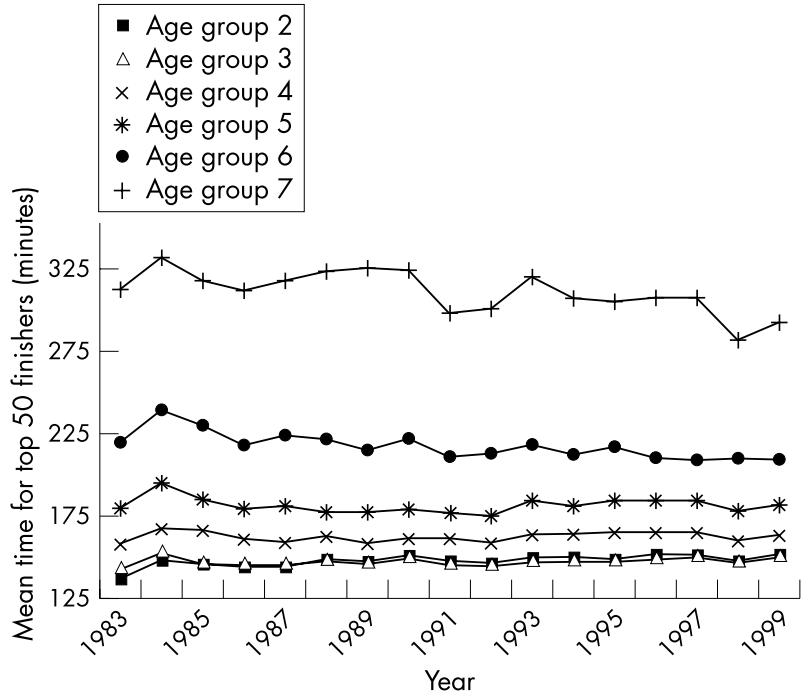

Figure 3 Mean running times of the top 50 male finishers in the NYC Marathon broken down by age group. Runners in age groups 2 and 3 showed a significant increase in running time $(p<0.001)$. Runners in age groups 5,6 , and 7 showed a significant decrease in running time $(p<0.001)$, with the greatest time improvement in age group 7 of 1.89 minutes a year.

have led to an increase in longevity. The comorbidity relating age to failing health has been reduced..$^{14}$ The multifactor health benefits of exercise have been made clear, and the myth that older people cannot complete marathons has been dispelled.

A study by Jokl and Jokl ${ }^{16}$ assessing the development of athletic records in track and field events have shown steady and, in part, predictable improvements in performance over the last century. In certain male running events, the rate of world record improvement has diminished, with some events having a relatively asymptotic statistical curve for record performance advancement. These data have been interpreted as indicating that there may be a finite limit to human performance capabilities. (The study at that time did not take into consideration genetic and pharmaceutical manipulations that could be used to enhance performance levels.) Women's performance improvements in all events are still progressing at a rapid rate, their record data still being in the "ascending" level in graphic illustrations. No female track events show a significant slowing in the progression of future records advancement. If we apply this theory of predicting performance improvements to our data on marathon running for both sexes, it is likely that there will be further improvement in running times in the master's age group. Furthermore, women appear to have greater potential than men for improving performance. ${ }^{16-21}$

Our data also show that there are many significant sex related differences in master athletes. Female marathon participation showed a much greater percentage increase in all age groups $(\mathrm{p}<0.05)$. The rate of increased participation over time for women is significantly greater than for men $(p<0.05)$. In 1983 the male to female ratio was approximately 6:1. Assuming that this linear rate continues, there will be equal participation of men and women in the NYC Marathon by the year 2007. Like male master athletes, women across a wider spectrum of age groups showed a time related improvement in their performance. Female runners in the 40-49, 50-59, and 60-69 age groups have shown a significant improvement in performance times over the last 17 years $(\mathrm{p}<0.05)$. Runners aged $50-59$ have improved their mean time at a rate of 2.08 minutes a year, and those aged 


\section{Information box}

- There are numerous studies describing the physiological and biological effects of aging on muscle. ${ }^{22-26}$ There are also data suggesting that activity modifies the decline in the function of muscle with aging.

- This study provides new data on performance and participation trends of master male and female athletes in an important sporting event. Participation in the NYC Marathon is increasing at a greater rate among master athletes than in other age groups. The performance times of master athletes have improved over the last 17 years. Women are outpacing men in both participation and performance.

60-69 at a rate of 3.79 minutes a year. The time improvement observed for older female athletes is substantially greater than that for their male counterparts.

The increase in participation and improvement in master women's running times outpace those of their male counterparts. In the past, social restrictions and perceptions may have inhibited women's ability to perform or maximise their training opportunities. For example, the first official female marathon in the Olympics was not until 1984. This probably explains the more rapid improvements in female marathon times. Like the findings for women's world record track performances, the potential for improvements by women will probably be greater than for their male counterparts in the foreseeable future.

The most significant trends noted in the top 50 finishers in the male category are for the age groups 60-69 and 70-79. Although there is a significant improvement for the male runners aged 50-59, a mean increase of eight seconds a year could be argued as being relatively stationary. If the top 100 finishers in each male category are examined, the same trends are seen, but a more notable 14 second mean improvement is noted in the 50-59 age group (data not shown). However, the most impressive improvements occur in the age groups 60-69 and 70-79 for men and 50-59 and 60-69 for women. It is also notable that in age group 70-79 for men and 60-69 for women, because of the lower participation in the 1980s, fewer than 50 participants were evaluated for some of the subsets of data. Despite the smaller group in those earlier years, we believe this represents an even more "elite" subset of runners who were able to run a marathon at that time, despite it not being "fashionable" to do so. Regardless, a significant trend over the last 17 years is noted for these age groups.

Assessment of the performances of the top 50 male and 50 female competitors in the NYC Marathon over the last 17 years leads to the following conclusions. Master athletes (50 years and older) are improving their performance at significantly $(p<0.01)$ greater rates than the younger participants. Senior women are improving their marathon running times at a greater rate $(\mathrm{p}<0.01)$ than senior men.

It is noteworthy that 1983 was an exceptional year for performances in the NYC Marathon. For almost every age group, running times outpaced many subsequent years. Historians of the NYC Marathon believe that the establishment of other autumn marathons in subsequent years led to a decrease in the number of world class runners participating in this marathon, but the exact reasons for the exceptional results remain incompletely understood. All the data presented in this paper include the 1983 times, and significant performance changes are nonetheless identified. However, the magnitude of the improvements would have been more impressive if this exceptional year had been excluded. These data were not excluded because no reason, such as a change in the course or environmental conditions, was identified.

This study used times for the NYC Marathon as a representation of trends seen in the population over the last two decades. Studies of other marathons, as well as other sporting events, may also characterise the underlying themes of increasing activity and performance of master athletes, as well as female participation in general.

\section{Authors' affiliations}

P Jokl, P M Sethi, A J Cooper, Department of Orthopaedics, Yale University, New Haven, CT, USA

\section{REFERENCES}

1 US Department of Commerce, Bureau of the Census. Statistical abstract of the United States: the national data book, 1998. 118th ed. Washington, DC: US Government Printing Office, 1998: 1-63.

2 US Department of Health and Human Services. Physical activity and health: a report of the Surgeon General. Atlanta: US Department of Healthand Human Services, Centers for Disease Control and Prevention, National Center for Chronic Disease Prevention and Health Promotion, 1996:3-13.

3 Coggan AR, Spina RJ, King DS, et al. Skeletal muscle adaptations to endurance training in 60- to 70-yr-old men and women. J Appl Physiol 1992:72:1780-6.

4 Fiatarone MA, $\mathrm{O}^{\prime} \mathrm{Neill} E F$, Solares $\mathrm{G}$, et al. Exercise training and nutritional supplementation for physical frailty in very elderly people. N Engl J Med 1994;330:1769-75.

5 Kavanagh T, Shephard RJ. The effects of continued training on the aging process. Ann N Y Acad Sci 1977;301:656-70.

6 Galloway M, Jokl P. Age and sports participation: the effect of aging on muscle function and athletic performance. Sports Medicine and Arthroscopy Review 1996:4:221-3.

7 Galloway MT, Jokl P. Aging succesfully. J Am Acad Orthop Surg 2000;8

8 Pyka G, Lindenberger E, Charette S, et al. Muscle strength and fiber adaptations to a year-long resistance training program in elderly men and women. J Gerontol 1994:49:M22-7.

9 Kirchner EM, Lewis RD, O'Connor PJ. Effect of past gymnastics participation on adult bone mass. J Appl Physiol 1996;80:226-32

10 Coggan AR, Spina RJ, Rogers MA, et al. Histochemical and enzymatic characteristics of skeletal muscle in master athletes. J Appl Physiol 1990;68:1896-901

11 Lexell J, Taylor CC, Sjostrom M. What is the cause of the ageing atrophy? Total number, size and proportion of different fiber types studied in whole vastus lateralis muscle from 15 - to 83 -year-old men. J Neurol Sci 1988;84:275-94.

12 Rogers MA, Hagberg JM, Martin WH III, et al. Decline inVO ${ }_{2}$ max with aging in master athletes and sedentary men. J Appl Physiol 1990;68:2195-9.

13 Pollock M, Foster C, Knapp D, et al. Effect of age and training on aerobic capacity and body composition of master athletes. J Appl Physiol 1987;62:725-31

14 Kries J, Crapo LM. Vitality and aging. San Francisco: WH Freeman \&Co, 1981:76-91.

15 Hagberg J, Allen W, Seals D, et al. A hemondynamic comparison of young and older endurance athletes during exercise. J Appl Physiol 1985;58:2041-6.

16 Jokl E, Jokl P. The physiological basis of athletic records. Springfield, IL: Charles C Thomas, 1968:86-103.

17 Allen WK, Seals DR, Hagberg JM, et al. Lactate threshold and distancerunning performance in young and older endurance athletes. J Appl Physiol 1985;58:1281-4.

18 American College of Sports Medicine. Position stand: the recommended quantity and quality of exercise for developing and maintaining cardiorespiratory and muscular fitness and flexibility in healthy adults. Med Sci SportsExerc 1998;30:975-91

19 American College of Sports Medicine. Position stand: exercise and physical activity for older adults. Med Sci Sports Exerc 1998;30:992-1008.

20 Bailey AJ, Robins SP, Balian G. Biological significance of the intermolecular crosslinks of collagen. Nature 1974;251:105-9.

21 Buckwalter JA, Lane NE. Athletics and osteoarthritis. Am J Sports Med 1997;25:873-81.

22 Booth FW, Weeden SH, Tseng BS. Effect of aging on human skeletal muscle and motor function. Med Sci Sports Exerc 1994;26:556-60.

23 Cartee GD. Aging skeletal muscle: response to exercise. Exerc Sport Sci Rev 1994;22:91-120.

24 Kirkendall DT, Garrett WE Jr. The effects of aging and training on skeletal muscle. Am J Sports Med 1998;26:598-602.

25 Larson EB, Bruce RA. Health benefits of exercise in an aging society. Arch Intern Med 1987; 147:353-6

26 Lexell J, Downham D. What is the effect of ageing on type 2 muscle fibres?[letter]. J Neurol Sci 1992;107:250-1. 